\title{
Research on Mobility Model of the VANET Based on the Environment of Avoidance
}

\author{
Yuan-Yuan Deng, Xiao-Qiang Xiao, Peng Zhou, Wei-Xun Ning \\ Computer School, \\ National University of Defense Technology, \\ Changsha (410073), Hunan, China \\ E-mail: blue0020101@163.com,xqxiao@nudt.edu.cn,1092873889@qq.com,ningweixun@nudt.edu.cn
}

\begin{abstract}
Due to the factors such as the high velocity of vehicles, the limited communication range of vehicles, the data transmission reliability and stability of the VANET (Vehicular Ad Hoc Network) is susceptible to traffic conditions and the influence of driving environment. So the mobility model of vehicles plays an important role in the routing protocol design and performance evaluation of VANET. In this paper, we proposed an IDM_A mobility model which takes the collision avoidance behavior of vehicles into consideration based on the characteristics of real vehicle track. After the simulation using the network simulator NS, the results verify the feasibility and effectiveness of the model proposed.
\end{abstract}

Keywords-VANET; mobility model; collision avoidance; network simulation

\section{INTRODUCTION}

Nowadays, cars have become an integral part of the modern society as a convenient transport. An increasing number of car ownership results many problems such as traffic jams and traffic safety in the city life. VANET(Vehicular Ad Hoc Network) is a no center, multiple hop and temporary autonomous system built by the short communication between vehicles, which can provide fast, efficient and powerful real-time traffic information, improve the security of driving environment, reduce the traffic accident casualties and ease the problem of traffic congestion.

Due to many factors such as economy, the difficulty of logic and technology limitations, it is difficult to evaluate VANET performance in the real environment, simulation is the best choice for the study of VANET and performance evaluation of proposed algorithms. In order to generate the approximate environment of VANET simulation, the appropriate vehicle mobility model must be defined to reflect the real track of vehicles.

In reality, there are some scenarios that the ordinary vehicles would avoid the special vehicle, the ordinary vehicles' tracks may change the original driving pattern, such as under the condition that an ambulance or a fire truck appears. Thus the performance of whole VANET routing would be impacted.
Unfortunately, most of the present VANET mobility models are based on a single type of vehicles ${ }^{[1-4]}$, and do not consider the avoidant trajectory while constructing the vehicle mobility model.

Therefore, based on the driver's following behavior, the VANET mobility model-IDM_LC ${ }^{[5]}$ (Intelligent Driver Model with Lane Change) is firstly expanded to IDM_A (Intelligent Driver Model with Avoidance) in this paper. To compare with the performance of these two kinds of model, GPSR routing algorithm with different models is simulated with NS-3. The data delivery ratio is selected as the metrics and how does the density of nodes affect the results are also analyzed in the end.

\section{THE VEHICLE MOBILITY MODEL_IDM_A}

In this paper, VANET communication scenarios consist of the ordinary vehicles and special vehicles (such as ambulance, fire truck, etc.) are considered. As shown in Figure 1, special vehicle ran in the mid-lane, the avoidance information is sent out. The ordinary vehicles in front of the special vehicle choose the strategy depending on the received signal and its current position: vehicles in the middle lane turn to the left or right lane under the condition of safety, and the vehicles in the lanes beside will slow down, to ensure that the vehicles changing lanes safely.

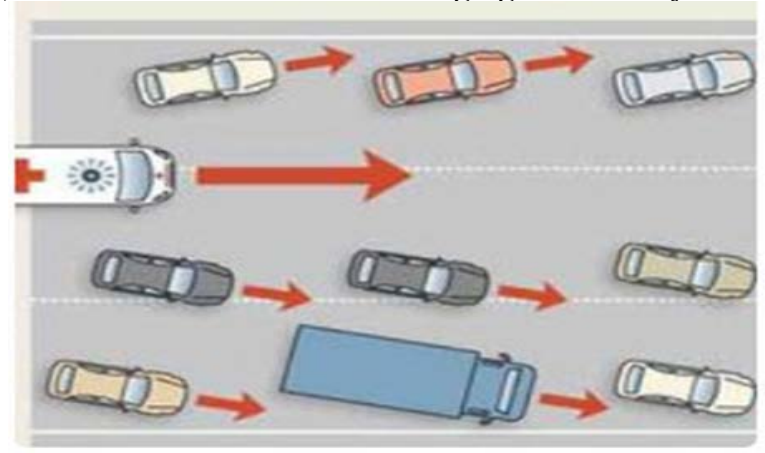

Figure 1. The scenario of vehicles' avoidance. 


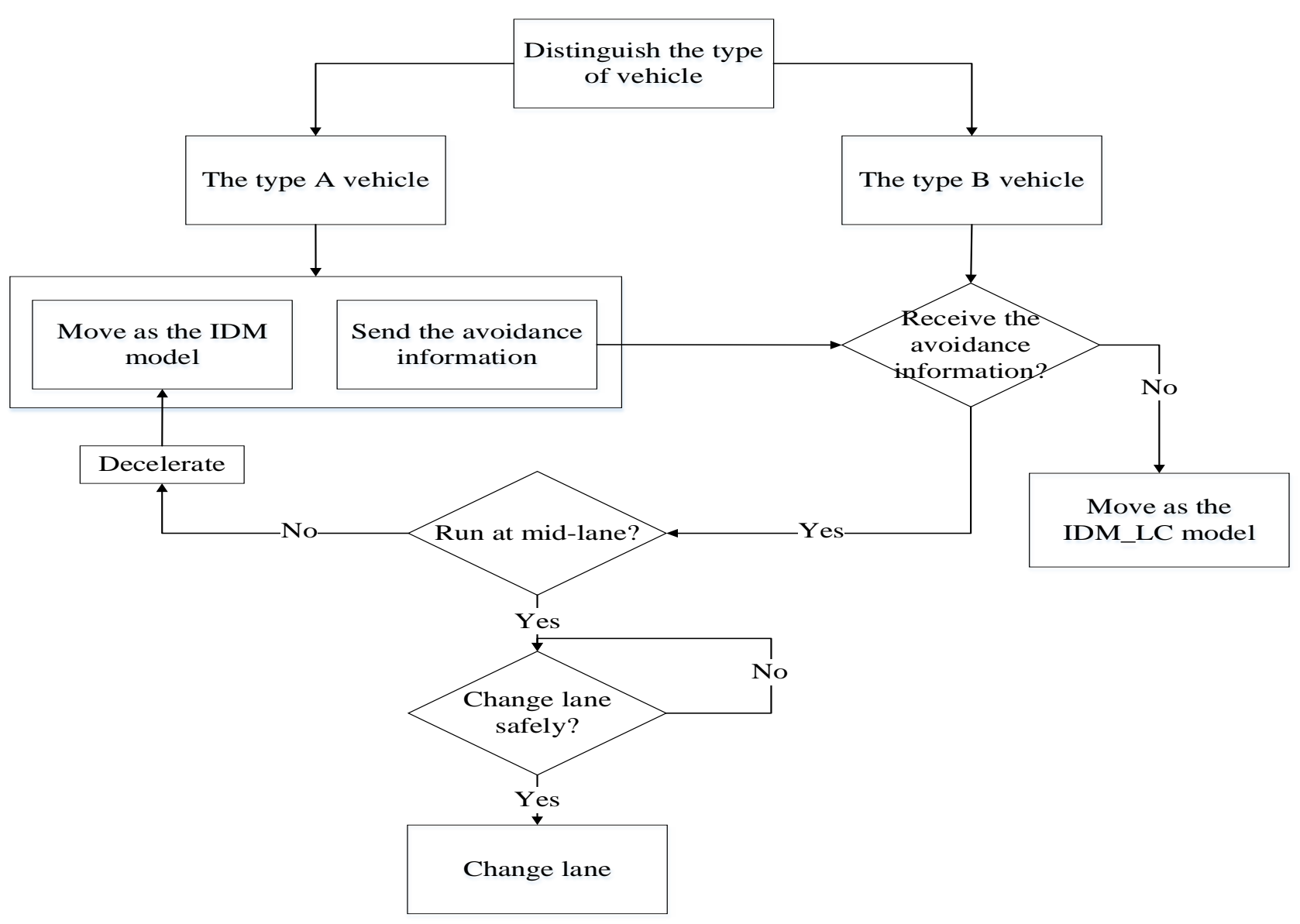

Figure 2. The structure of IDM_A.

IDM_LC is a kind of mobility model developed from $\mathrm{IDM}^{[6]}$ (Intelligent Driver Model) which can reflect the pattern of vehicle driving well. Our mobility model IDM_A is developed from IDM_LC by means of the vehicle classification and establishing communication, thus the avoidant driving behavior could be stimulated if regard the packets as the avoidant signal.

\section{A. IDM_LC Mobility Model}

IDM model only considers the following behavior of the driver, which means the vehicle's velocity is influenced by the velocity of the vehicle in front. And IDM_LC mobility model use a model called MOBIL ${ }^{[1]}$ to control the vehicle changing the lane.

Although IDM_LC mobility model can well reflect the changing lane and overtaking the drivers' behavior, it can't simulate the behavior of avoidance. There are two reasons:

- Same as most other mobility models, in the IDM_LC mobility model, all nodes are in a state of equality, and obey the same mobility rules. That is, IDM_LC does not distinguish the priority for vehicles.

- For IDM_LC, the center of the model is the current vehicle. By collecting the current state information (such as distance, position, velocity, acceleration, etc.) of the vehicles nearby to imitate the natural driving behavior, the model can predict the future state of current vehicle. But it does not take account of the distance communication among the vehicles. So, it can only effectively reflect the driving behavior based on the feedback of the traffic state in the driver's view. And it can't reflect drivers respond to the environment events (such as collision avoidance signal, siren, etc.) or the long-distance transmission information.

\section{B. IDM_A Mobility Model}

In order to solve the two problems of IDM_LC shown above, Intelligent Driver Model with Avoidance (IDM_A for short) based on IDM_LC is proposed and its structure is shown in Figure 2.

In IDM_LC, vehicles are divided into two kinds of different types, namely type A (special) and type B (ordinary). We assume that every node can received special vehicle avoidant signals through wireless communication such as Wi-Fi.

The behaviors of these two kinds of vehicles are described as follows:

1) A type vehicles(special): Type A vehicle only run 
in the safe driving lane (say mid-lane), the vehicle keeps its own speed, and never change the lane except that there is some obstacles. It will broadcast avoidant information to imitate the emergency vehicle siren signals. From the view point of mobility model, it will move follow the IDM model.

2) B type vehicles(ordinary): When there is no type A vehicle in the scenario, type $B$ vehicle will move according to the IDM_LC mobility model. Once some vehicles receive avoidant information and know the existence of type $A$ vehicle, these vehicles have to make the way for type A vehicle under some safe condition such as slow down and turn to other lanes. The example scenario is shown in Figure 3.

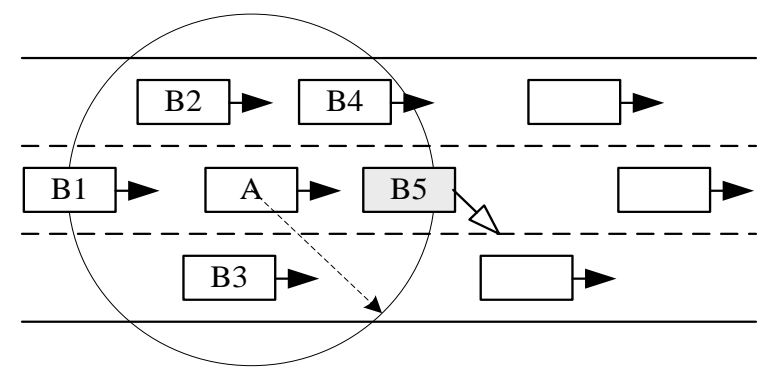

Figure 3. Scenario of avoidance.

In this scenario, $\mathrm{A}$ is the emergency vehicle (type A), a circle describe its communication ability. B1 to B5 are ordinary cars (type B) in the communication rage of A. At this time, $\mathrm{B} 5$ in the mid-lane received the avoidance information and wants to change the lane. B5 and the type B vehicles in the lane beside will slow down, to ensure the safety during changing the lane.

\section{THE SIMULATION AND NUMERICAL ANALYSIS}

\section{A. Simulation Scenario}

The IDM_LC mobility model has been implemented with NS-3, providing a new module called "vanet-highway"[7]. It combines the mobility model and network simulator successfully into a single simulator, implements the embedded simulation architecture. Since VANETs demand the instantaneity of traffic safety and traffic efficiency information communication, simulation of the new mobility model must get some accuracy to some distance.

Our simulator will follow the architecture of vanet-highway. The simulation scenario is also extended at the same time. Some details about setting up the simulator are listed as follows:

- The simulation scenario is extended by a single and one-way road. To simulate avoidant behavior, the lane width is $5 \mathrm{~m}$, the length is $1000 \mathrm{~m}$ long and the road has three lanes. The coordinates of lane expressed by the ordinate, from top to bottom were $10.0,5.0,0.0$. The middle lane (ordinate 5.0 ) is a safe lane for emergency vehicles.

- Vehicles are divided into two types. Vehicle ID of Type A (emergency vehicle) is negative, while vehicle ID of type $B$ is positive.

- To implement the avoidant message broadcasting and forwarding, the node should add new function that could get packet and store it.

To implement the vehicle avoidance, the key processing procedure is shown in Figure 4.

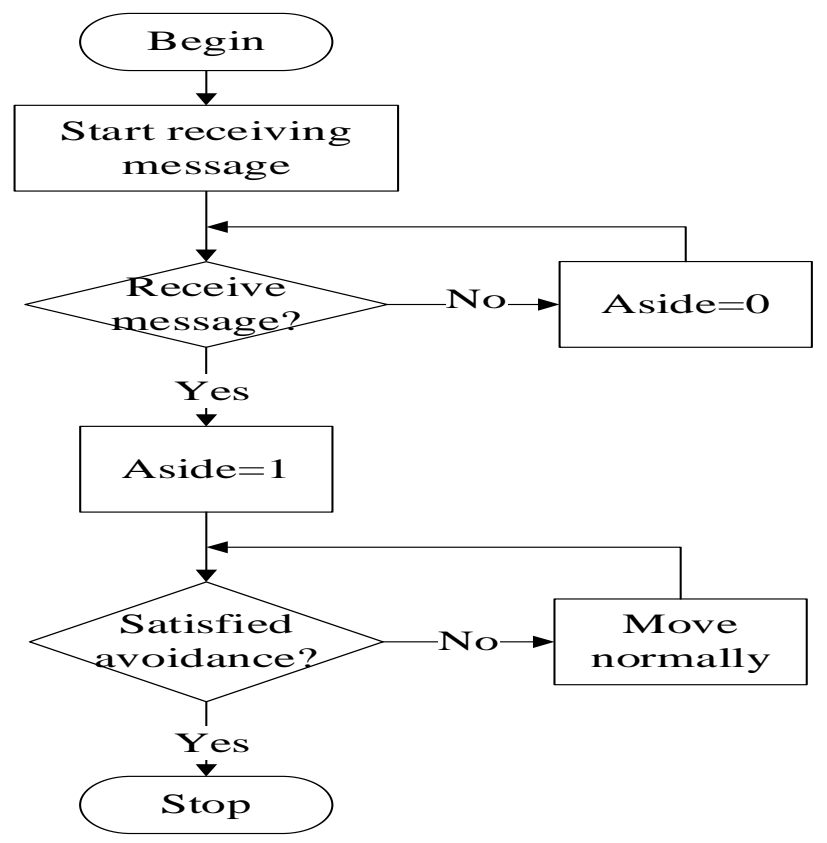

Figure 4. The processing procedure of avoidance.

A flag called "Aside" show the condition of changing lane, when "Aside" is zero, the current vehicle doesn't need to change lane. For example, the current vehicle is behind the emergency vehicle, even in the middle lane and within the range of the signal communication, it doesn't need to change the lane. When "Aside" equal to one, current vehicle should change lane at this time in the case of safety.

In NS-3, after compiled by "waf" and run the program, file "vehicleTrace.csv" which records the vehicle moving behavior and file "networkTrace.csv" which stores the communication messages among vehicles are generated. Results of these two files can be visualized by the software "vehicle viewer".

\section{B. Experiment Result Analysis}

In order to verify the effectiveness of IDM_A mobility model and the feasibility of VANET simulation architecture, the routing algorithm GPSR ${ }^{[8]}$ is selected to compare the communication performance under both kinds of mobility model scenarios. The packet delivery ratios under different traffic density are collected and analyzed.

Some experiment scenario parameters as shown in Table 
TABLE I. TABLE THE SIMULATION PARAMETER SETTINGS

\begin{tabular}{|c|c|}
\hline Simulation parameter & Value \\
\hline Size of scenario & $6000 \mathrm{~m} \times 3000 \mathrm{~m}$ \\
\hline Vehicle flow & $0.5,1,3,6,8$ \\
\hline Communication radius & $200 \mathrm{~m}$ \\
\hline Time & $1500 \mathrm{~s}$ \\
\hline Size of packets & $512 \mathrm{~kb}$ \\
\hline Rate of packets & 1 packet/s \\
\hline $\begin{array}{c}\text { Special vehicles join time } \\
\text { interval }\end{array}$ & $\begin{array}{c}\text { Random(join the group of special } \\
\text { vehicles into the mid-lane, each group } \\
\text { contains three special vehicles) }\end{array}$ \\
\hline
\end{tabular}

After simulate 20 times and get the average of data, the delivery ratios of the different models are shown in Figure 5 .

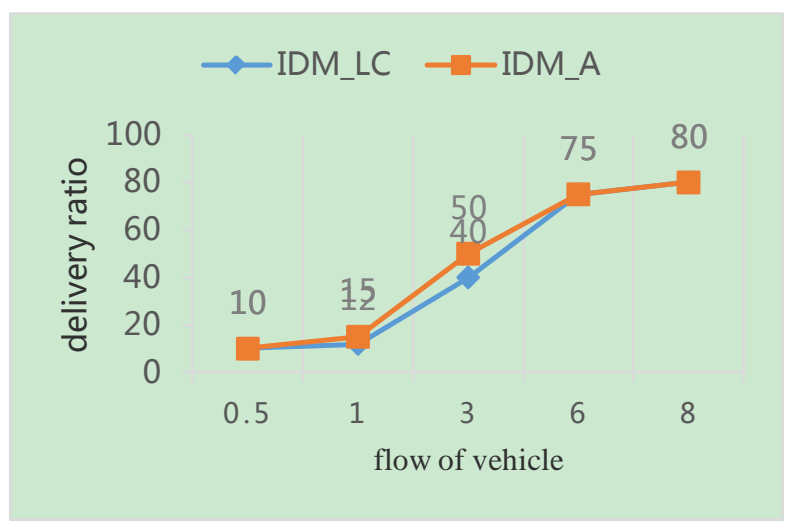

Figure 5. The data delivery ratio.

While the traffic density is increasing, for both models, packet delivery ratio has been improved. The experiment results show that in the case of low density, the data delivery ratio of IDM_LC is lower than IDM_A; when the density is high, the result is opposite. The gap between these two results is not big, but for the VANET which is mainly used in intelligent traffic, the effect is serious, especially under the emergency signals.

When the traffic density is sparse, even if there are emergency vehicles joining the scenario, vehicle trajectory would not change too much, so the delivery ratios under two models have no difference.

Because GPSR is a kind of greedy algorithm ${ }^{[8-9]}$, it chooses the closest node to the destination within the range of communication as the next-hop node. When the traffic density increases, the number of ordinary vehicles which change lane on the road increases, making the packets lost easily. When traffic density is intensive, the vehicle in the lane beside should slow down in order to ensure the safety. Thus the density in other lanes increase and the velocity of the whole scenario, and the number of neighbor nodes also increase. So GPSR algorithm in IDM_A mobility model has higher delivery ratio. When the traffic density increase to a certain value, the lane reach in a congestion state, the traffic conditions are similar in both two models, the addition of special vehicle will not cause obvious delivery ratio gap.

\section{CONCLUSION}

VANET and corresponding technology have important application prospects, and their performance evaluation methods are mainly through simulation. Mobility model plays an important role in the simulation work. Most nowadays VANET mobility models typically define the vehicles into the same status, and they cannot well reflect the avoidant driving behaviors of the vehicles which will greatly influence the density and the velocity of the vehicle. In this paper, we presents IDM_A mobility model which divides the vehicles into two types and considers the real vehicle moving features of different type. Choosing the GPSR routing algorithm, the simulator using NS-3 is built up and the message delivery ratio is analyzed for the performance comparing of IDM_LC and IDM_A. The experimental results are discussed under different traffic density, showing the feasibility and the effectiveness of the proposed model.

Due to the limitation of objective factors, there are still some difference between the model and the real scenario. Further consideration will focus on the effectiveness of avoidance behavior under different routing algorithms.

\section{ACKNOWLEDGMENT}

This research is supported by the National Science Foundation of China under Grant No. 61272485.

\section{REFERENCES}

[1] Wei Da, Wang Yanxi, Wang Jian, Liu Yanheng, Deng Weiwen. A survey on mobility models of vehicular Ad Hoc networks. Chinese Journal of Computers. 2013, 36(4): 677-700.

[2] Vinícius F.S. Mota, Felipe D. Cunha, Daniel F. Macedo, José M.S Nogueira, Antonio A.F. Loureiro. Mobility models and tools in opportunistic networks:A survey. Computer Communications. 2014(48):5-19.

[3] C. Mascol, M. Musolesi, Designing mobility models based on social network theory. ACM SIGMOBILE, 2010, 11(3):59-70.

[4] C. Bettstetter. Smooth is better than sharp: a random mobility model for simulation of wireless networks. ACM International Workshop on Modeling, 2002:19-27.

[5] J. Haerri, F. Filali, C. Bonnet, Marco Fiore. VanetMobiSim: generating realistic mobility patterns for VANETs. Proc. $3^{\text {rd }}$ ACM International Workshop on Vehicular Ad Hoc Networks. LosAngels, USA, 2006:96-97.

[6] M. Treiber, A. Hennecke, D. Helbing. Congested traffic states in empirical observations and microscopic simulations. Physical Review E Statistical Physics, 2000, 62(2A):1805-1824.

[7] D. Bradley. Improvements in VANET simulator in NS-3. Master's Project Final Report, Department of Computer Science, Old Dominion University, 2011.

[8] B. Karp, H.T. Kung. GPSR: greedy perimeter stateless routing for wireless networks. MOBICOM 2000, Boston, MA, USA. 243-254.

[9] B.T. Sharef, R.A. Alsaqour, M. Ismail. Vehicular communication ad hoc routing protocols: A survey. Journal of Network and Computer Applications. 2014, 40(1):363-396. 